\title{
Semiquantitave Characterization of Pigments by EDS
}

\author{
Marcela Miyagusuku ${ }^{1 *}$, Ana Lucía Marzocca ${ }^{1}$, Patricia Bozzano ${ }^{1}$, Adriana Dominguez ${ }^{1}$, \\ Gonzalo Zbihlei ${ }^{1}$ \\ ${ }^{1}$ Constituyentes Atomic Center of National Commission of Atomic Energy (CAC- \\ CNEA), Electron Microscopy Division, San Martín, Buenos Aires, Argentina.
}

*Corresponding author: $\overline{\text { miyagusu@cnea.gov.ar }}$

Semiquantitative characterization by EDS (Energy Dispersive Spectroscopy) was performed in different color pigments. The studies were conducted by a scanning electron microscopy (SEM), FEI SEM Quanta 200 and EDAX Apollo in Scanning Electron Microscopy Laboratory of Material Science Management Group at Constituyentes Atomic Center of National Commission of Atomic Energy (CACCNEA).

The scope of this work is the proposal of a methodical procedure, that will allow in the future, obtained a semiquantitative protocol for EDS analysis of pigments. Sample preparation, electron accelerating voltage, current beam/size spot, spectrum acquisition parameters regarding type of the detector (detector time-constant, dead time (DT) and collection time) as well as spectrum analysis and semiquantitative composition measurement were evaluated [1-3].

Several pigments were analyzed as part of a cultural heritage study, which include organic and inorganic samples. Simple stoichiometric between two elements and multielemental non-stoichiometric composition were found in inorganic samples. Figure 1 shows different samples of pigments that were prepared for SEM observation. As an example, the EDS spectrum obtained from one of the red color pigment ("Muestra 8") is shown in Figure 2.a. When DT was high (DT77) because of the increasing throughput (Figure 2.b.), the peak width also increased and peak shifting was also detected.

Based on our measurements, the first approximation of a reliable study, DT must be considered as a critical parameter and should be optimized when experimental conditions are set. 


\section{References:}

[1] Genesis Spectrum - User's Manual, EDAX INC., (2006) p. 5-53.

[2] The British Standards Institution BS ISO 15632:2012, BSI Standards Limited, (2012) p. 6-9.

[3] J. I. Goldstein et al. in "Scanning Electron Microscopy and X-Ray Microanalysis", Springer $4^{\text {th }}$ Edition, (2018), p. 461-469.

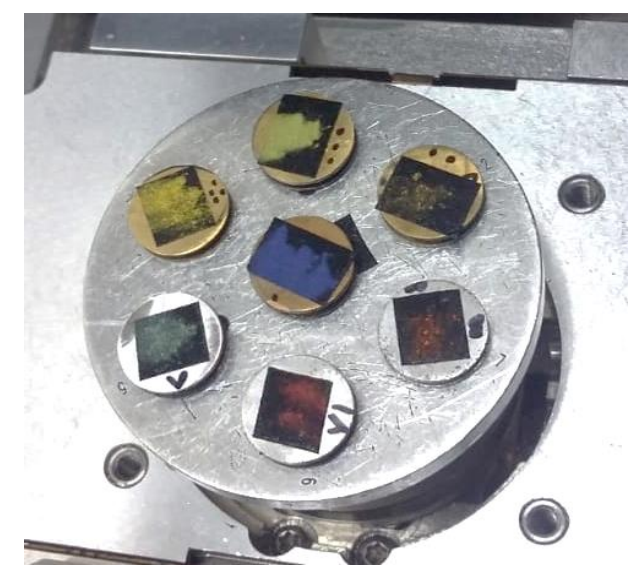

Figure 1: Photo of the pigments located in the sample holder of the SEM.

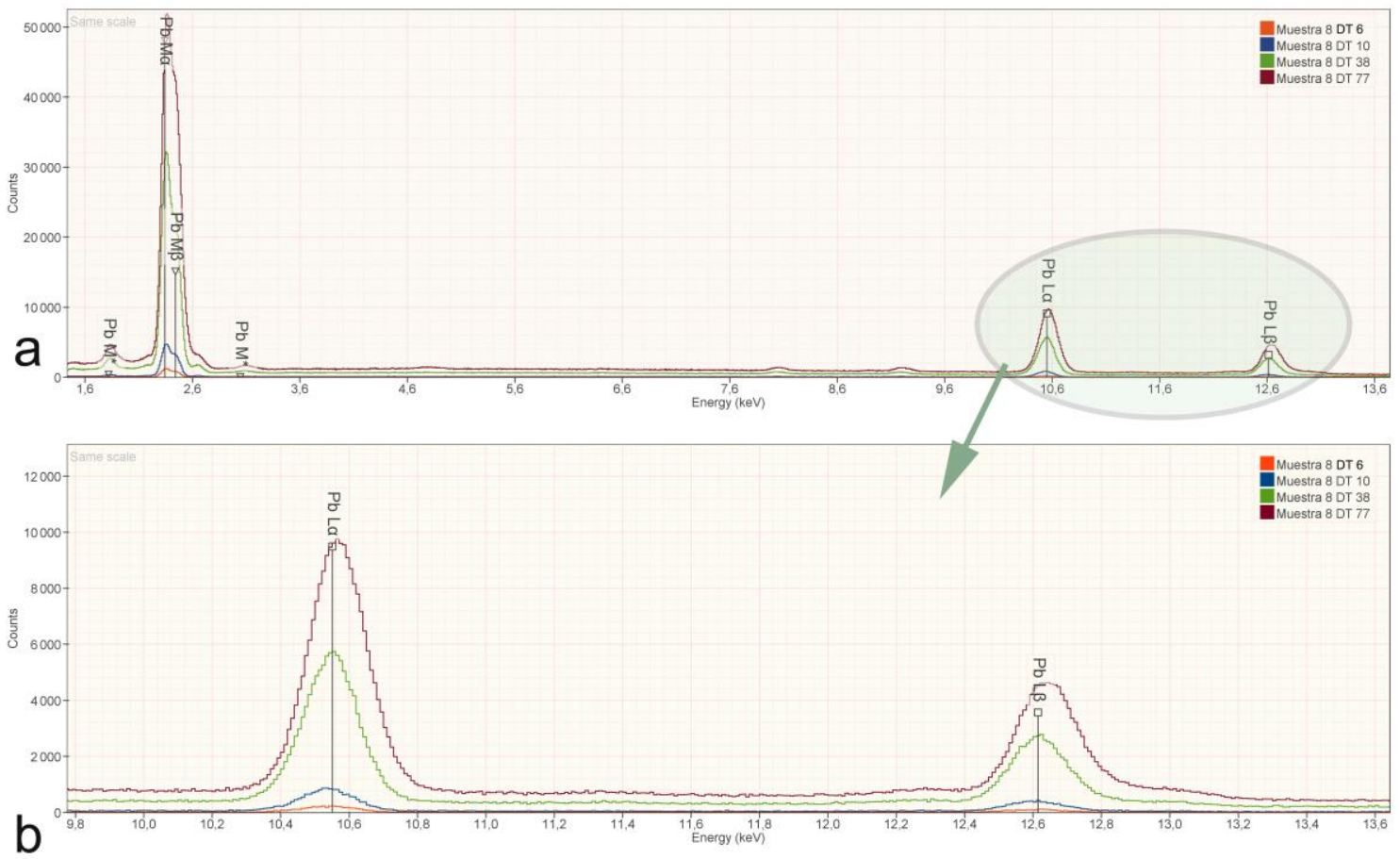

Figure 2: a. EDS spectrum obtained from one of the red color pigment. b. Approach of the EDS peaks marked with a circle 